\title{
Thermal Magnetic Behavior of Nanocrystalline Fe73.5Cu1Nb3Si13.5B9 Ribbons
}

\author{
Ang Ding ${ }^{1,2}$, Jie Hao ${ }^{1 *}$, JingHui Huang ${ }^{1}$, KunMing Qian ${ }^{1}$, Song $\mathrm{Ji}^{1}$, YanSong \\ Zhang ${ }^{1}$, MingLi Li ${ }^{1}$ and XingLong Dong ${ }^{2}$ \\ ${ }^{1}$ Inner Mongolia Metals Institute, 199 Lingyun Road, Ningbo, 315103, China \\ ${ }^{2}$ School of Material Science and Engineering, Dalian University of Technology, Dalian, 116024, \\ China \\ *1 hao jie82@163.com
}

\begin{abstract}
Keywords: Nanocrystalline Alloy, Thermal Magnetic Behavior, Exchange Coupling Interaction, Initial Permeability, Saturation Magnetization.
\end{abstract}

\begin{abstract}
Thermal magnetic behavior and microstructure of $\mathrm{Fe}_{73.5} \mathrm{Cu}_{1} \mathrm{Nb}_{3} \mathrm{Si}_{13.5} \mathrm{~B}_{9}$ ribbon annealed at different temperatures were investigated. It was indicated that the onset crystallization temperature of the ribbon was around at $530{ }^{\circ} \mathrm{C}$ and its Curie temperature can be enhanced from $320{ }^{\circ} \mathrm{C}$ to $385{ }^{\circ} \mathrm{C}$ by annealing. X-ray diffraction structural analysis showed that a single a-FeSi crystalline phase with grain size about $12 \mathrm{~nm}$ was precipitated from amorphous matrix for $\mathrm{FeCuNbSiB}$ ribbons annealed between $530{ }^{\circ} \mathrm{C}$ and $580{ }^{\circ} \mathrm{C}$. The $\alpha$-FeSi phases were octahedral shape confirmed by the morphology simulation and the AFM method. The thermal magnetic behaviors were complicated, as the $\mu_{\mathrm{i}}$ can be divided into two modes during increasing temperature, but for the Bs and Ms shown a normal decrease with increasing temperature. The coercive force $\mathrm{Hc}$ and remanence ratio $\alpha$ have shown a continuous monotonic decline in single-phase ribbon with increasing temperature, and increased to a max value or a peak and then decreased to zero in biphase ribbons. The thermal behaviors of magnetic properties were controlled by the exchange coupling interaction between the $\alpha$-FeSi grains and the intergranular amorphous phase. It is important to maintain an efficient exchange coupling interaction between $\alpha-\mathrm{FeSi}$ grains and the residual amorphous matrix, and it is necessary to sustain the higher Curie temperature for the application of the $\mathrm{FeCuNbSiB}$ ribbons in high temperature by controlling the size and crystallization degree of $\alpha$-FeSi grains.
\end{abstract}

\section{Introduction}

With the unique duplex ultrafine structure of nanocrystalline and amorphous phases, the nanocrystalline soft magnetic alloys successfully achieve excellent performances [1-4], such as high saturation flux density, high permeability, and low loss. Nevertheless, the magnetism of nanocrystalline soft magnetic alloys are sensitive to exoteric temperature, and can be lost completely as the temperature exceeds Curie temperature (Tc)[5]. Studying on the thermal behaviors can be obtain necessary information of the nanocrystalline magnetic alloys for the potential applications. Due to the two-phase structure with quite different Curie temperatures, the effective magnetic anisotropies and saturation magnetizations, the thermal behavior of nanocrystalline soft magnetic materials are complicated and affected by many factors [6-8]. Kulik and Hernando [9] investigated the relationship between the crystallization annealing temperature $(\mathrm{Ta})$ and the coercive force $(\mathrm{Hc})$ of Fe73.5Cu1Ta3Si13.5B9 nanocrystalline alloy in the temperature range from $480 \mathrm{oC}$ to $580 \mathrm{oC}$ and found that a maximum value (Tk) existed at Hc-T curve and changed with the annealing temperature. Hernando proposed [10] a two-phase structure of phenomenological theory to explain this phenomenon. Yanzhong Zhang [11] studied the thermal behavior of permeability on the nanocrystalline alloy, and made a qualitative interpretation for the experimental results.

In this paper the influence of annealing temperature on the structure and thermal magnetic properties of Fe73.5Cu1Nb3Si13.5B9 nanocrystalline alloy have been investigated. The thermal magnetic properties after a thermomagnetic treatment are characterized by the quasistatic hysteresis loops concerning its certain shapecoercivity, , saturation magnetization, remanence and so on. The 
structures of nanocrystallines have been investigated using X-ray diffraction in transmission geometry. The correlation of structure and magnetic properties of the nanocrystalline alloy has been analyzed and discussed.

\section{Experimetal}

Cast ingot of the soft magnetic alloy with nominal composition of Fe73.5Cu1.0Nb3.0Si13.5B9 (in at.\%) was obtained by arc melting of pure metals $\mathrm{Fe}, \mathrm{Cu}, \mathrm{Nb}, \mathrm{Si}$ and $\mathrm{Fe}-\mathrm{B}$ alloy (the purities of all raw metals are higher than $99.8 \mathrm{wt} \%$, and Fe-B alloy is $99.9 \% \mathrm{wt} \%$ ) under pure Ar atmosphere. The amorphous ribbons with about $10 \mathrm{~mm}$ in width and $30 \mu \mathrm{m}$ in thickness were prepared by a single-roller melt spinning method using above alloy ingot. The phases of as-quenched and annealed samples were examined by X-ray diffraction (XRD) using D/max-2500/PC with CuKa radiation. The microstructure of the nanocrystalline soft magnetic alloy was evaluated using atomic force microscope. Afterwards these ribbons were wound into toroidal core samples of $12 \mathrm{~mm}$ inner diameter and $20 \mathrm{~mm}$ outer diameter. To obtain the characteristic nanocrystalline structure, these toroidal samples were submitted to isothermal annealing at temperatures between 100 and $580 \mathrm{oC}$ for $0.5 \mathrm{~h}$ under the protection of hydrogen atmosphere. Then the initial magnetic permeability of the samples before and after the thermal treatment was measured by the impact method with $\mathrm{H}$ of $0.08 \mathrm{~A} / \mathrm{m}$. The DC magnetic properties were measured by the CL6-1, and the saturation magnetization (Ms), saturation magnetic flux density (Bs) and coercivity (Hc) were attained from the DC hysteresis loop, and the rectangle ratio $\mathrm{Br} / \mathrm{Bs}$ also been calculate by the datum.

\section{Results}

\section{Microstructure Characteristics}

\section{X-ray Diffraction}

In order to analyses the microstructure of annealed Fe73.5Cu1Nb3Si13.5B9 ribbons, XRD experiments are conducted and shown in Fig. 1. It is well know that the as-quenched ribbon, a typical amorphous phase, exhibited abroad scattering peak in the diffraction curve. While the patterns of the annealed samples show the characteristic (110), (200), (211), (220) and (311) diffraction peaks of a-FeSi crystalline phase and (200) diffraction peaks of Fe3Si crystalline phase, indicating that all the annealed samples have crystallized partially and their microstructure consists of the residual amorphous phase, the a-FeSi crystalline phase and a little Fe3Si crystalline phase. The content of crystalline phase increase accordingly with the increase of annealing temperature, and the intensity of diffraction peaks become stronger, as shown in the Fig. 1.

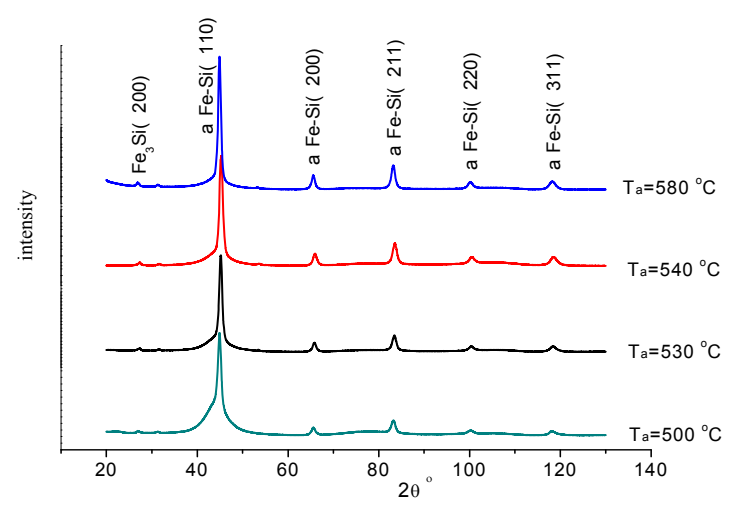

Figure 1. XRD patterns of annealed $\mathrm{FeCuNbSiB}$ alloy

From the X-ray diffraction structural analysis, crystal lattice constant of a-FeSi (a0), the content of $\mathrm{Si}$ in a-FeSi, average grain size of a-FeSi (da), the degree of short-range order $\delta$, average grain size of amorphous phase (dA), and t can be estimated by means of the Scherrer formula, as shown in Table 1. 
It can be seen that the da of the annealed $\mathrm{FeCuNbSiB}$ ribbons are about $12 \mathrm{~nm}$. Though there exist a little difference in grain size between the annealed samples, the grains size are small enough to randomly average out the local magneto crystalline anisotropies, and achieve an excellent magnetic softness according to Herzer'srandom anisotropy model [12].

Table 1. Micro-structural parameters of the $\mathrm{FeCuNbSiB}$ ribbons obtained by $\mathrm{X}$-ray diffraction structural analysis

\begin{tabular}{llllllll}
\hline & \multicolumn{7}{c}{$\alpha \mathrm{Fe}(\mathrm{Si})$} \\
No. & $\mathrm{Ta}\left({ }^{\circ} \mathrm{C}\right)$ & $\mathrm{a}_{0}(\mathrm{~nm})$ & $\mathrm{Si}(\mathrm{at} \%)$ & $\mathrm{d}_{\alpha}(\mathrm{nm})$ & $\delta(\mathrm{nm})$ & $\mathrm{d}_{\mathrm{A}}(\mathrm{nm})$ & $\mathrm{t}(\mathrm{nm})$ \\
\hline 1 & 500 & 0.283715 & 20.49399 & 9.5 & 2.672675 & 0.25346 & 1.5166 \\
2 & 520 & 0.283415 & 21.82911 & 13.5 & 2.716663 & 0.25161 & 2.2996 \\
3 & 530 & 0.28303 & 23.5425 & 12.4 & 2.409599 & 0.25267 & 1.3258 \\
4 & 540 & 0.283654 & 20.76547 & 10.6 & 2.782814 & 0.24921 & 1.0537 \\
5 & 580 & 0.283557 & 21.19715 & 14.3 & 2.749161 & 0.25149 & 1.3575 \\
\hline
\end{tabular}

\section{X-ray Topography Analysis of Nanocrystals}

To achieve further information the internal structure of the $\mathrm{FeCuNbSiB}$ ribbons is investigated by the morphology simulation method according to popa theory [13] and the AFM technique. The morphology simulation result and the AFM micrographs foe the sample annealed at $580 \mathrm{oC}$ for 40 min are shown in Fig. 2. In Fig. 2a the simulation image of the nanocrystalline shows octahedral shape. Fig. $2 b$ is the AFM morphology of the surface of the ribbon which agrees with the positions predicted from the simulation image. The bright yellow field images are due to the strong force between the sample surface and the probe, especially for the up part of nanocrystalline (the up part of the octahedron as shown Fig. 2a). The AFM results confirme the high percentage of crystallization and the crystallite size measured by the X-ray diffraction structural analysis. It should be observed that the average crystallite size determined by the XRD analysis (table. 1) is usually lower than the grain size observed in AFM (the da the annealed FeCuNbSiB ribbons are about $14 \mathrm{~nm}$ from the Fig. $2 \mathrm{~b})$. The grains in the annealed samples contain sub-grains or dislocation cells which are separated by the low-angle grain boundaries [14]. The grain size obtained from the X-ray diffraction structural analysis is equal to the average size of the domains which scatter X-rays strongly. As a result, the XRD differentiates between sub-grains and dislocation cells, even if the miss-orientations are very difficult and small.
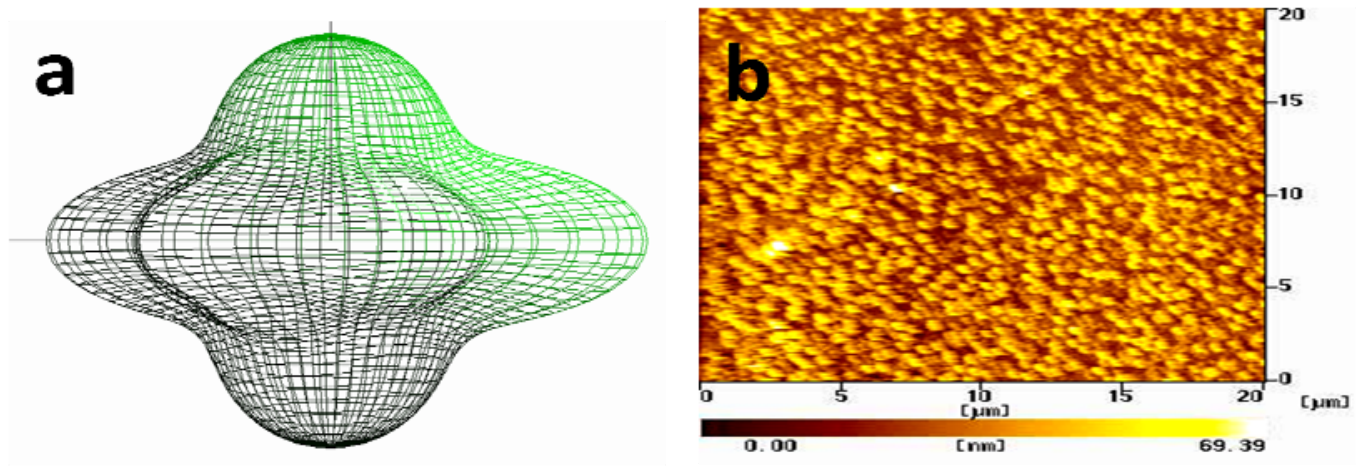

Figure 2. Microstructure of the FeCuNbSiB ribbons: a) morphology simulation by the Popa theory, b) the AFM micrographs of the sample annealed at $580{ }^{\circ} \mathrm{C}$ for $40 \mathrm{~min}$ 


\section{Magnetic Properties}

\section{Thermal - Saturation Magnetization Curve}

Fig. 3 presents the dependence of the Ms of the annealed samples at different temperature. The Ms display a slight variation with different treat condition, except for the sample treated at $520{ }^{\circ} \mathrm{C}$. The curies temperature $\left(\mathrm{T}_{\mathrm{c}}\right)$ of the sample treated at $520{ }^{\circ} \mathrm{C}$ is lower than that of others samples obviously. This result is induced by fellow reasons. Firstly, the different can be responsible for the different crystallization degree in the samples with various treatments. Secondly, the increase of treat temperature accelerates the crystallization of ribbons, and result in an increase in grain volume fraction, which in turn leads to an increase in the curies temperature. Due to an increase in grain volume fraction, this can reduce the effective magnetic moment by change the magnetic exchange interactions of the magnetic neighbor grains. It is worth mentioning that the $\mathrm{Ms}$ of the samples decrease with the increase of the temperature. But with the increase of treat temperature, which induces the increase of grain volume fraction, the decrease of the Ms became slower until to the curies temperature.

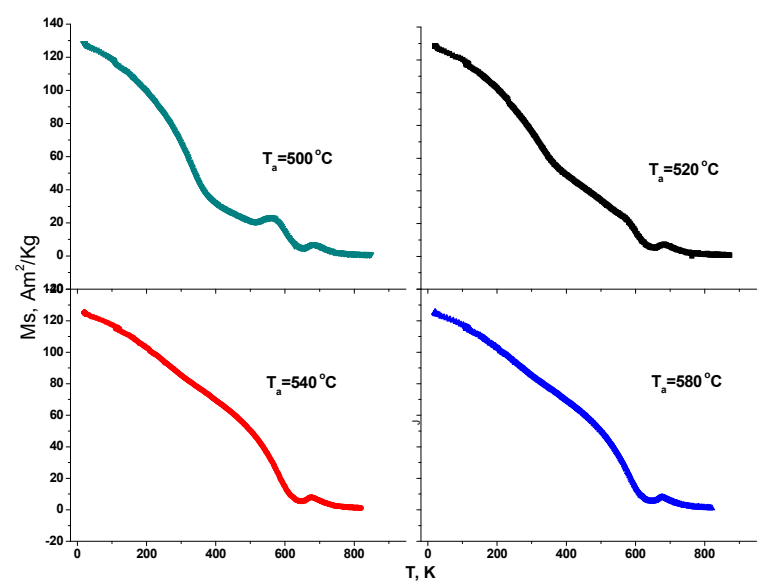

Figure 3. Ms of the annealed samples at different temperature

\section{The Thermal Behavior of the Initial Permeability}

In order to characterize the high-temperature magnetic response as well as the Curie temperature of the $\mathrm{FeCuNbSiB}$ ribbons, the temperature dependence of initial permeability ( $\mu_{\mathrm{i}} \mathrm{-T}$ curves) was measured. Fig. 4 showed the $\mu_{\mathrm{i}}$-T curves of as-quenched samples and the annealed ribbons (from 100 ${ }^{\circ} \mathrm{C}$ to $580{ }^{\circ} \mathrm{C}$ ). On all $\mu_{\mathrm{i}}$-T curves a characteristic sharp Hopkinson peak is observed at the Curie point of the amorphous phase. Fig. 4a showed the $\mu_{\mathrm{i}}$-T curves of as-quenched ribbons, which is due to the faster decrease of magnetic anisotropy than that of annealed ribbons. It can be easily seen that an obvious $\mathrm{T}_{\mathrm{c}}$ for the samples, which annealed less than $500{ }^{\circ} \mathrm{C}$, is around $320{ }^{\circ} \mathrm{C}$. When the annealing temperature exceeded to $500{ }^{\circ} \mathrm{C}$, the $\mathrm{T}_{\mathrm{c}}$ for the samples became more difficult to identify, indicating that the nanocrystals play an important role to change and enhance the $\mathrm{T}_{\mathrm{c}}$ for annealed ribbons.

The above results implied that, when the annealing temperature is low, only one low temperature amorphous phase Hoppkinson peak is appeared, indicating that under low annealing temperature the ribbons remains the single-amorphous phase structure, but it exist structural differences between the as-quenched samples and the low treat temperature annealed ribbons. With increasing annealing temperature, the Curie temperature of the amorphous phase is increased, and the width of the Curie peak is broadened (the temperature between the peak and the end point of peak temperature). When the annealing temperature reached $500 \mathrm{oC}$, besides the amorphous phase Hoppkinson peak, the crystalline phase Hoppkinson peak of $\alpha$-FeSi phase also appeared in the $\mu \mathrm{i}-\mathrm{T}$ curves with a double Hoppkinson peak characteristic. The results are similar with the Fan's researches [15]. So the result descript that the $\alpha$-FeSi phase has precipitated from the matrix, and compose biphase structure with the residual amorphous phase, which has been proven by X-ray diffraction results. With further 
increasing annealing temperature, the Hoppkinson peak width of right branch curve continues to increase, but the Curie temperature of the amorphous phase has shown a downward trend. When the annealing temperature was higher than $540 \mathrm{oC}$, the permeability decrease with increasing temperature, only appear $\alpha-F e S i$ phase Hoppkinson peak at high temperature, and amorphous phase Hoppkinson peaks is disappeared. But X-ray diffraction results indicate that the annealed ribbons still remaine a little of amorphous phase at this stage.

Taking these findings, it can be found the thermal initial permeability behavior of nanocrystalline soft magnetic material has diversity, and was divided into single and two Hoppkinson peaks or other two types. For the $\mathrm{FeCuNbSiB}$ ribbons, their transformation temperature was in range of $530 \mathrm{oC}-540$ oC.
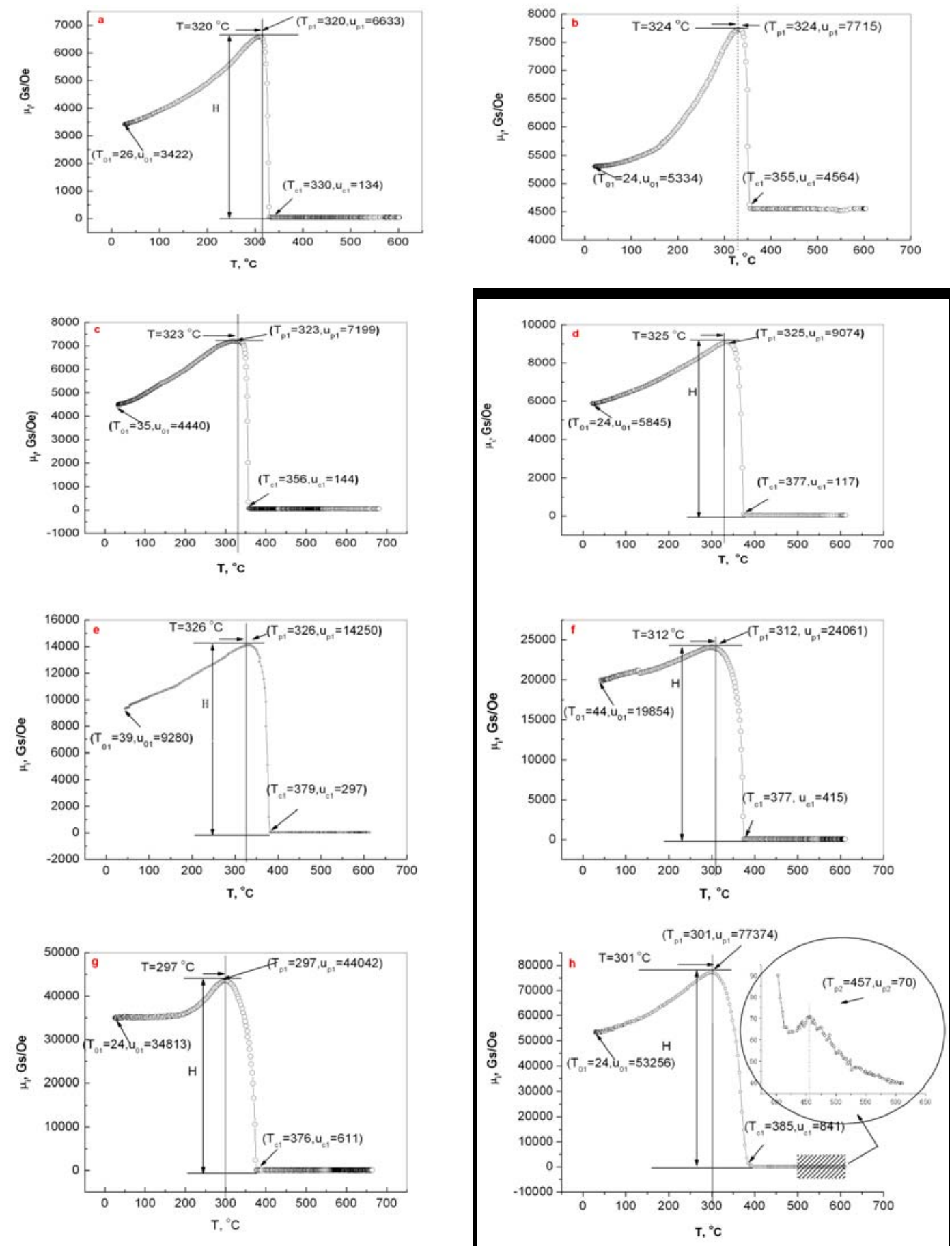

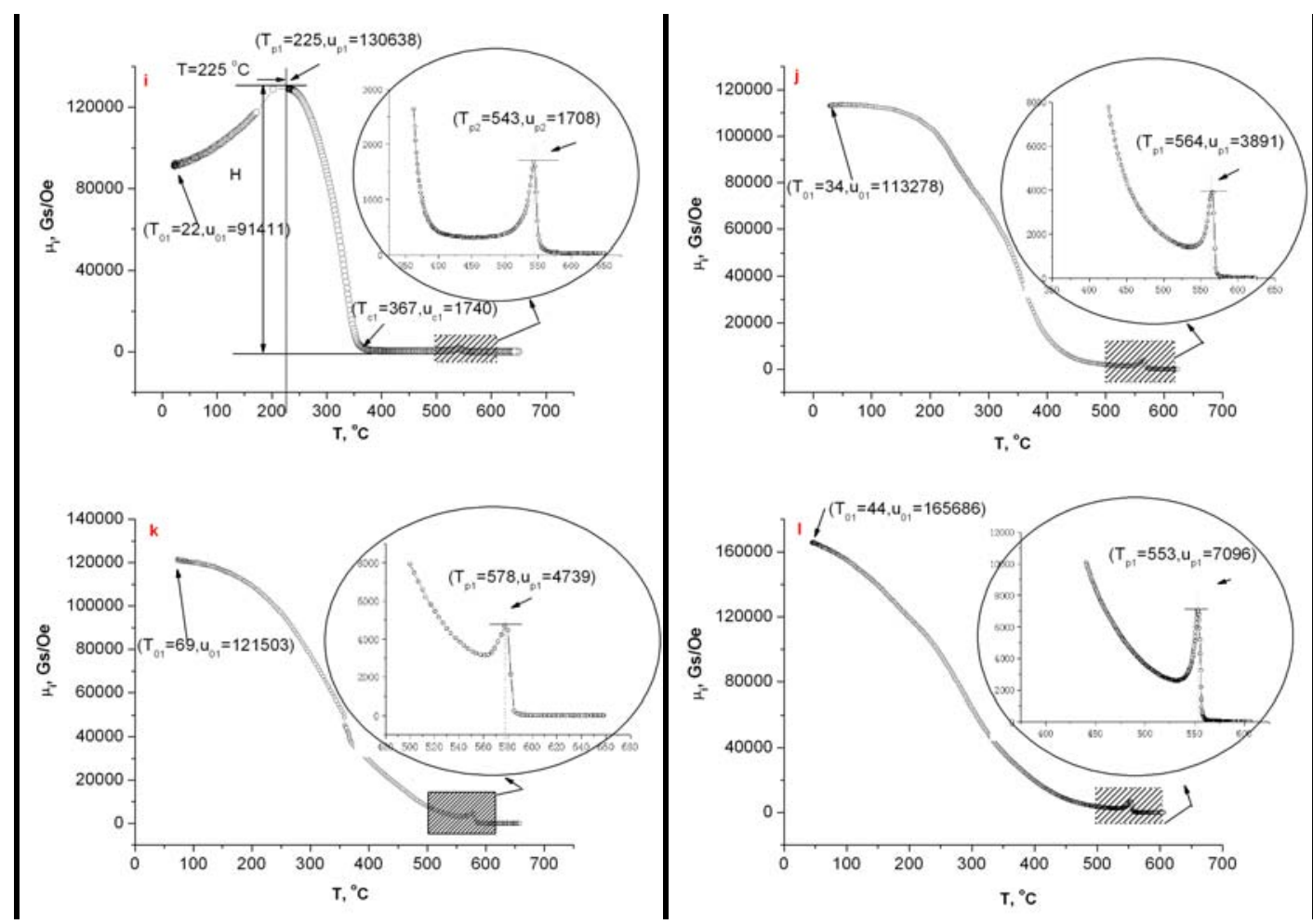

Figure 4. Thermal behavior of the initial permeability for the $\mathrm{FeCuNbSiB}$ ribbons with different treatment: (a) as-quenched; (b) $100{ }^{\circ} \mathrm{C}$; (c) $200{ }^{\circ} \mathrm{C}$; (d) $300{ }^{\circ} \mathrm{C}$; (e) $350{ }^{\circ} \mathrm{C}$; (f) $400{ }^{\circ} \mathrm{C}$; (h) $500{ }^{\circ} \mathrm{C}$; (i) $520{ }^{\circ} \mathrm{C}$; (j) $540{ }^{\circ} \mathrm{C}$; (k) $560{ }^{\circ} \mathrm{C}$; (l) 580 ${ }^{\circ} \mathrm{C}$

\section{Thermal Behavior of Saturation Magnetic Flux Density}

In Figure 5, the dependences of saturation magnetic flux density $(\mathrm{Bs})$ on temperature for the annealed $\mathrm{FeCuNbSiB}$ ribbons at $800 \mathrm{kA} / \mathrm{m}$ are shown. According to the experiment results, the Bs at the room temperature shows an increasing trend with increasing annealing temperature, from $0.8 \mathrm{~T}$ of the amorphous ribbon to the $1.2 \mathrm{~T}$ of the ribbon treated at $530{ }^{\circ} \mathrm{C}, 540{ }^{\circ} \mathrm{C}$, and $580{ }^{\circ} \mathrm{C}$. It is considered that the crystalline phase ( $\alpha-\mathrm{FeSi}$ phase) induced the increase of the Bs for the ribbons treated above the $530{ }^{\circ} \mathrm{C}$. From the figure, the Bs thermal behaviors of the ribbons have shown the similar change trend with each other. The saturation magnetic flux density Bs decrease with the increase of temperature, and decrease to zero around the Curie temperature like the permeability thermal behaviors. The reason will be discussed in the next parts of the paper in detail.
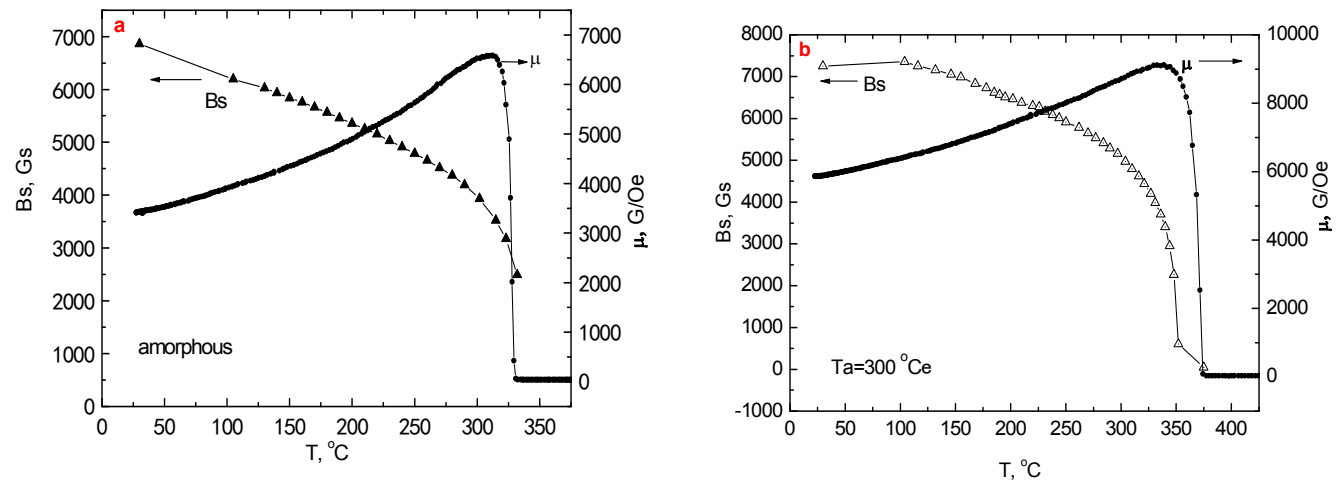

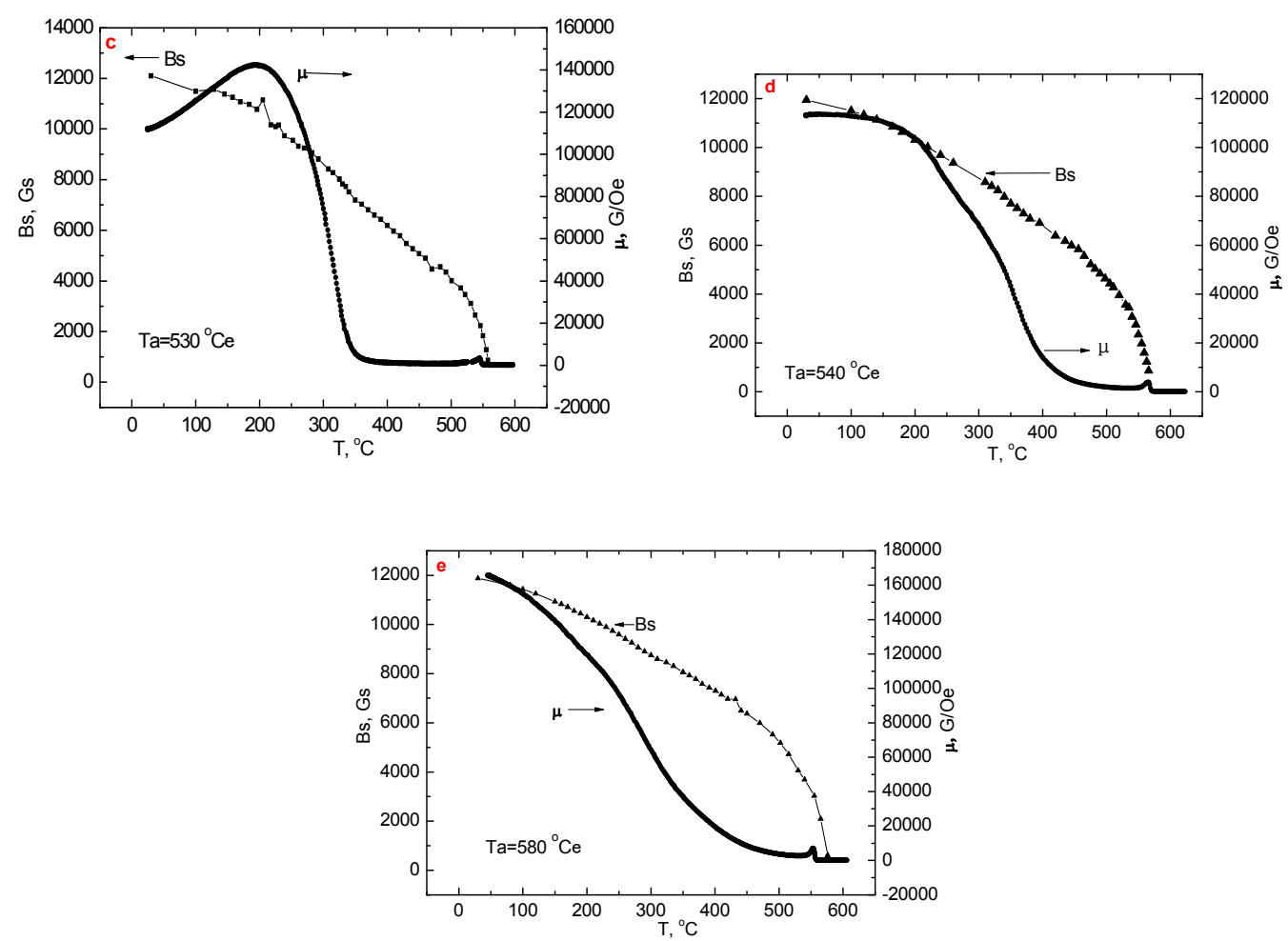

Figure 5. Bs of the annealed samples at different temperature

\section{The Coercivity and Remanence Ratio Thermal Behavior}

The thermal behavior of coercivity and remanence ratio $(\alpha)$ of the ribbons were measured from the room temperature to the temperature above the Curie temperature, and shown in fig. 6. For single-phase soft magnetic amorphous ribbon (as shown in fig. 6a and b), the coercive force Hc and remanence ratio $\alpha$ have shown a continuous monotonic decline with increasing temperature. When the temperature rose to the peak of amorphous phase Hoppkinson and the zero point's temperature of the Bs, namely the Curie temperature, the coercive force $\mathrm{Hc}$ and the remanence ratio $\alpha$ are reduced to zero, and the ribbons loss magnetic properties, which similar to the results Hernando ${ }^{[10]}$ studied. For biphase nanocrystalline ribbons (as shown in fig. 6c-f), When the temperature rose to the peak of amorphous phase Hoppkinson and the zero point's temperature of the Bs, namely the Curie temperature, the coercive force $\mathrm{Hc}$ and remanence ratio $\alpha$ also reduce to zero, which similar to that of the amorphous ribbon. But in the process of increasing temperature, there exist very different behaviors between the amorphous ribbon and the annealed ribbons. From the figure 6 , it is clear that the coercive $\mathrm{Hc}$ and remanence ratio $\alpha$ firstly increased to a max value or a peak and then decreased to zero with the increase of temperature, but this phenomenon didn't occurred in the amorphous ribbon. The peaks of Hc-T curves and $\alpha$-T curves are occurred in the temperature range, in the range the permeability $\mu$ change rapidly, indicating that the peaks of $\mathrm{Hc}$ and $\alpha$ are related to the ferromagnetic-paramagnetic transition in the ribbons during increasing temperature. Another phenomenon can be found from the figure, the peak of Hc did not coincide with the peak of peak $\alpha$ in the position of temperature, and the temperature at the peak of $\mathrm{Hc}$ is higher than that of the $\alpha$, implying that there exist different mechanism in the thermal behavior of Hc and $\alpha$. 

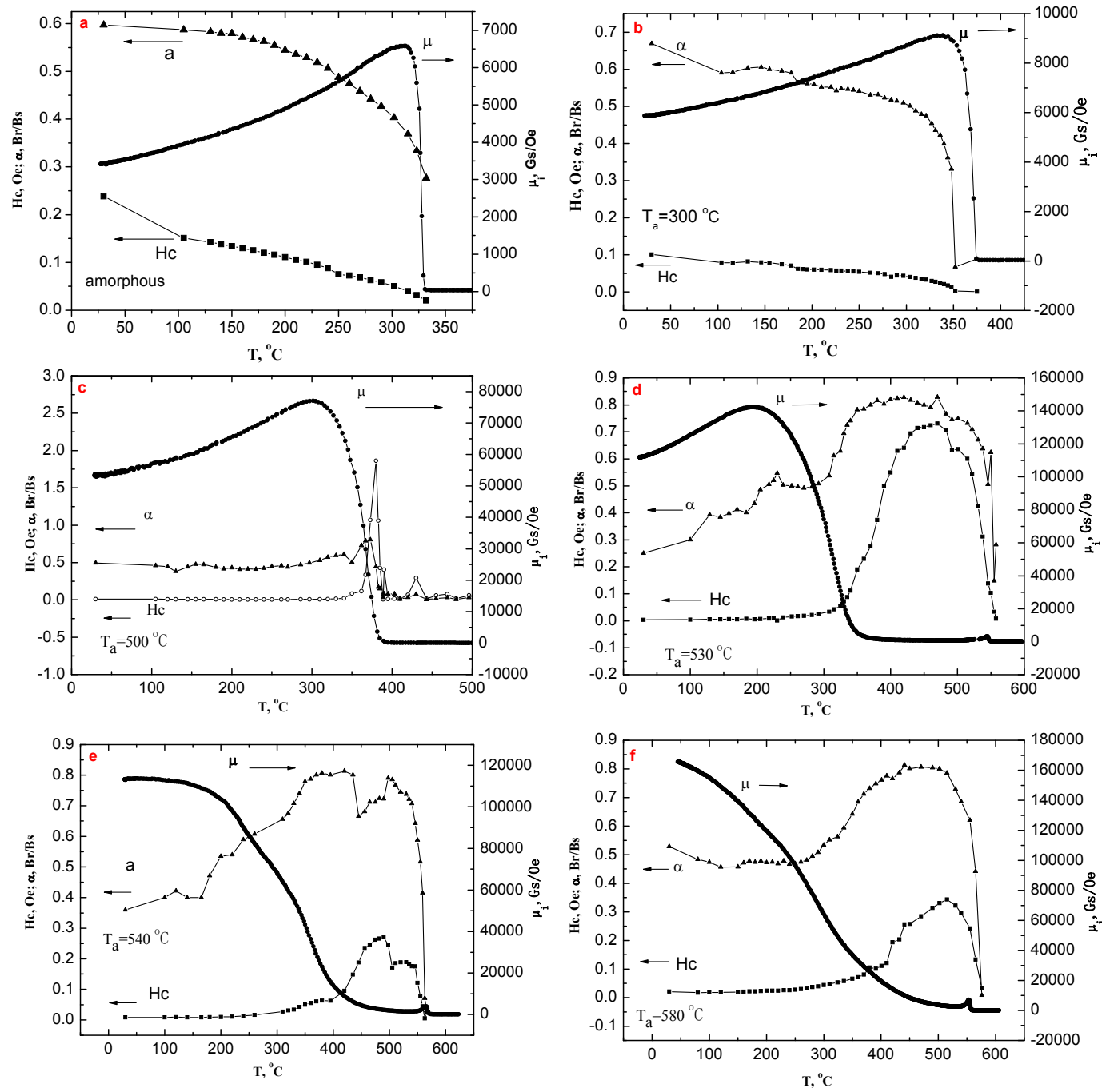

Figure 6 . The temperature curve of coercivity and remanence ratio

\section{Discussions}

\section{Analysis of the Super Paramagnetic Properties of $\alpha$ Fe-Si Nanocrystals}

From above results, it can be conclude that the microstructure of the FeCuNbSiB nanocrystalline ribbons have a stronger effect on the thermal behavior magnetic properties. From the X-ray diffraction structural analysis, the $\mathrm{FeCuNbSiB}$ nanocrystalline ribbons contain the nano- $\alpha \mathrm{FeSi}$ grains and amorphous phase. According to the magnetic theory, when the ultrafine magnetic $\alpha \mathrm{FeSi}$ grains are isolated by the amorphous phase, the ribbons will exhibit a superparamagnetic characteristic. In the superparamagnetic stage, there is no interaction between the nano- $\alpha \mathrm{FeSi}$ grains, when the magnetic anisotropy energy of the grain equal to thermal motion energy of the grain, the thermal activation energy may be able to overcome the magnetic anisotropy barrier, this induce that the magnetization vector of the ultrafine grain will no longer along the easy magnetization direction of the grains, but ceaselessly back and forth reverse between the different easy magnetization directions. So the magnetic properties of ribbons have show very different between the amorphous ribbon and the annealed ribbons. But for the superparamagnetic theory, there exist a critical grain size $\left(D_{c}\right)$ for the $\mathrm{FeCuNbSiB}$ ribbons, and the $\mathrm{D}_{\mathrm{c}}$ can be calculated by the following formula 1 :

$$
D_{\mathrm{c}}=\sqrt[3]{\frac{600 k T}{\pi K_{1}}}
$$


Where $\mathrm{k}$ is Boltzmann's constant; $\mathrm{T}$ is the absolute temperature; $\mathrm{K} 1$ is the magnetic anisotropy constant. If the grain size of the ribbons is smaller than the superparamagnetic critical grain size Dc, superparamagnetic characteristic will appears and the remanence and coercivity of the ribbons tends to zero, and need a high magnetization field to make the ribbons approaching the saturation magnetization.

The critical grain size Dc of the $\alpha$-FeSi grains in the $\mathrm{FeCuNbSiB}$ ribbons at different $\mathrm{T}$ are calculated and shown in Figure 7. In the Figure the Da is the average grain size of a-FeSi calculated from the X-ray diffraction structural analysis. It can be easily seen that the average size of a-FeSi grain is much smaller than the grain size of the critical grain size Dc, indicating that the average grain size of a-FeSi has to meet the superparamagnetic presentation condition. In fact, the $\mathrm{FeCuNbSiB}$ ribbons at room temperature $(\mathrm{T}=25 \mathrm{oC})$ does not appear the superparamagnetic characteristic for above results, the superparamagnetic characteristic were suppressed, which is directly related to the exchange coupling mechanism in nanocrystalline soft magnetic materials [16].

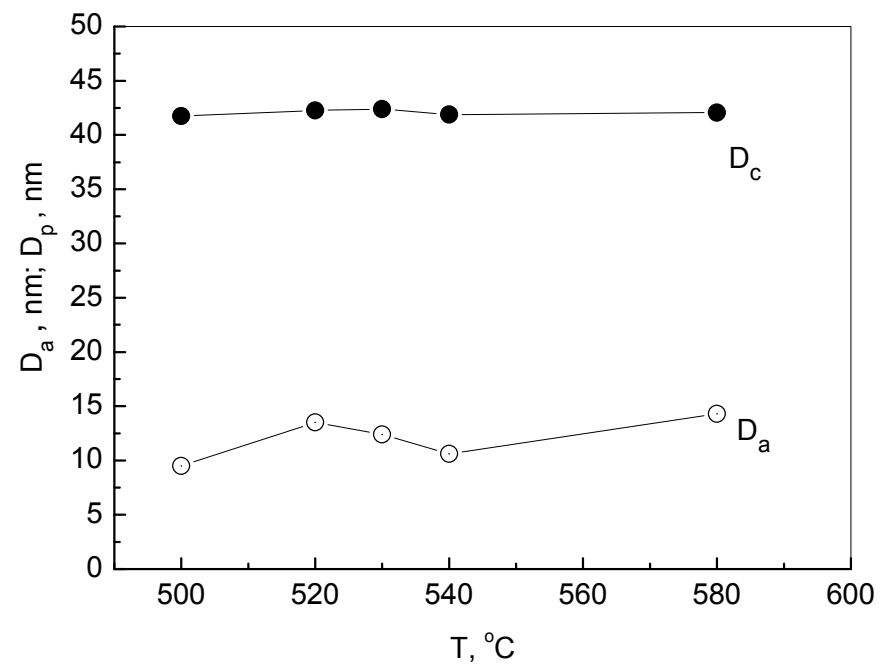

Figure 7. The critical grain size $\mathrm{D}_{\mathrm{c}}$ of the $\alpha \mathrm{Fe}(\mathrm{Si})$ grains in the FeCuNbSiB ribbons

\section{Analysis of the Exchange Coupling Mechanism of $\alpha$ Fe-Si Nanocrystals}

Using the amorphous random anisotropy Alben model, Herzer [17] successfully explained the origin of the excellent soft magnetic properties in the nanocrystalline soft magnetic material. He pointed out that when the $\alpha-\mathrm{FeSi}$ grain size is less than the exchange coupling critical length De, the adjacent crystal grains will have exchange coupling effect, so that the local anisotropy of the crystal grains is effectively averaged out, and make the effective magnetic anisotropy of the nanocrystalline $\mathrm{FeCuNbSiB}$ ribbons to an extreme low level, therefore, the $\mathrm{FeCuNbSiB}$ ribbons possess high soft magnetic properties. For the nanocrystalline soft magnetic material, the De can be regarded as the critical exchange coupling length of about $35 \mathrm{~nm}$, greater than $\alpha-\mathrm{FeSi}$ grain size D $\alpha$ of 10-20nm (table 1). The De can be calculated by the following formula 2 :

$$
D_{a}<D_{\mathrm{e}}=\sqrt{A_{a} / K_{a}}
$$

The theory exchange coupling critical length proposed by Herzer is establish on the basic of Alben's single phase amorphous random anisotropy model, which is different with bipolar structure of nanocrystalline soft magnetic materials. In the nano-crystalline soft magnetic materials, the $\alpha$-FeSi exchange coupling interaction between grains, in fact, are carried out and transferred by the amorphous phase between $\alpha-\mathrm{FeSi}$ grains, as shown in the figure 8 . So researching the exchange coupling interaction of the $\alpha$-FeSi grains should consider the affect of the magnetic properties of the $\alpha-\mathrm{FeSi}$ phase, in addition to considering the affect of the amorphous phase. 
Indeed, for biphase structure, R. C. O. Handiey[18] expound in its monograph that when the distance between grains (t) is less than the exchange-coupled grains critical size Lex, the magnetization from one grain to the other grains is carried out by penetrating through the grain boundary phase, so the exchange coupling interaction between the $\alpha-\mathrm{FeSi}$ grains must penetrate through the intergranular amorphous phase [19].

Compared with the exchange coupling critical length De, for the nanocrystalline soft magnetic ribbons, the Lex not only is relate to the $\mathrm{A}$ and $\mathrm{K}$ of $\alpha$-FeSi phase, but also associated with the difference of saturation magnetization $(\delta \mathrm{Ms})$ between the $\alpha$-FeSi phase and the amorphous phase, which relate to the duplex microstructure parameters of nanocrystalline $\mathrm{FeCuNbSiB}$ ribbons. When $\delta \mathrm{Ms}$ reduce to zero, namely the saturation magnetizations of $\alpha$-FeSi phase are equal to that of the amorphous phase, the Lex reach to the maximum, and the ribbons exhibit excellent magnetic properties. K.Y He consider [20] that when the saturation magnetization is equal in the grain phase and the amorphous phase, the equal induce the origin of excellent magnetic properties in the nanocrystalline $\mathrm{FeCuNbSiB}$ ribbons, for which there is need for further research.



Figure 8. Schematic diagram of nanocrystalline soft magnetic materials exchange coupling[21]

The Lex can be calculated by the formula 3:

$$
L e x=\sqrt{A /\left[K_{u}+2 \pi\left(M_{s}^{\alpha}-M_{s}^{A}\right)^{2}\right]}
$$

Where $\mathrm{A}$ is exchange integration constant; $\mathrm{Ku}$ is the magnetic anisotropy constant; ${ }^{M}{ }_{s}^{\alpha}$ is the saturation magnetizations of $\alpha \mathrm{FeSi}$ phase; ${ }^{M}{ }_{s}^{A}$ is the saturation magnetizations of amorphous phase. According to the formula 3, the Lex of nanocrystalline $\mathrm{FeCuNbSiB}$ ribbons at different temperature are calculated and shown in Figure 8. It is observe that the thickness t of the soft magnetic amorphous phase is smaller than the exchange-coupled grains critical size Lex, indicating that the exchange coupling interaction between the $\alpha$-FeSi grains penetrate through the intergranular amorphous phase in the nanocrystalline $\mathrm{FeCuNbSiB}$ ribbons, which play important roles to improve the performance of the soft magnetic materials.

In the formula 3 , note that the $\mathrm{A}, \mathrm{K},{ }^{M_{s}^{\alpha}}, M_{s}^{\mathrm{A}}$ all are the temperature variables, indicating the Lex is a temperature variables too, which will affect the thermal behavior of the nanocrystalline $\mathrm{FeCuNbSiB}$ ribbons. So further analyzing the relation between the Lex and the temperature is very significant to explore its effect on the magnetic thermal behavior of the ribbons. If the $\mathrm{K}$ « $\mathrm{M}$, the formula 3 can be simplified to the formula 4 :

$$
\operatorname{Lex}=\sqrt{A / 2 \pi \Delta M^{2}}
$$

Where $\triangle \mathrm{M}$ is the difference of the ${ }^{M_{s}^{\alpha}}, M_{s}^{A}$. By the magnetic theory, the A=Ms, and in order to maintain consistency with the test magnetic thermal behavior, the Ms is instead by Bs, and then the formula 4 can be further simplified to the formula 5 : 


\section{$\operatorname{Lex} \propto \Delta M \sqrt{M_{s} / 2 \pi}$}

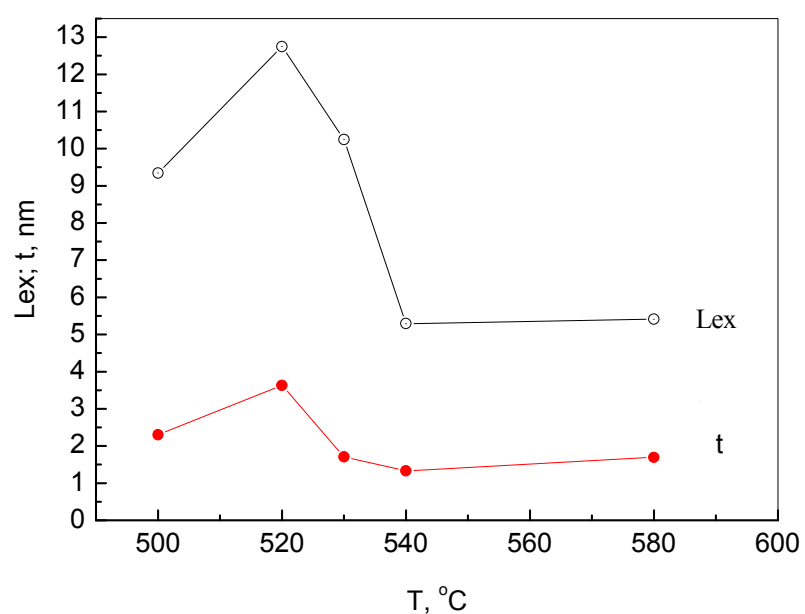

Figure 9. Lex and th of the nanocrystalline $\mathrm{FeCuNbSiB}$ ribbons at different temperature

The Lex is calculated with formula 5 and shown in Figure 9. It can be found the Lex has a peak or valley during increasing temperature, and the temperature of peak or valley close to the characteristic temperature of $\mu, \mathrm{Hc}, \alpha$. when Lex decreases with increasing temperature, the Hc and $\alpha$ exhibit an opposite change with increasing temperature, indicating the decrease of Lex induce the distance between grains $(\mathrm{t})$ is larger than the exchange-coupled grains critical size Lex. Therefore, the exchange coupling interaction between the $\alpha$-FeSi grains are weak, and the effective anisotropy are strong relatively. At the lower temperature area, the Hc and $\alpha$ change slightly with increasing temperature, indicating the exchange coupling interaction between the $\alpha$-FeSi grains are sustained at steady state; but at the higher temperature area, the exchange coupling interaction between the $\alpha-\mathrm{FeSi}$ grains are broken or weaken, inducing the decrease of the Hc and $\alpha$ with increasing temperature.

Through the above analysis, it is clear the exchange coupling interaction is a very important aspect specific for the nanocrystalline $\mathrm{FeCuNbSiB}$ ribbons. In the ribbons exchange coupling interaction between the $\alpha$-FeSi grains mainly occurs via the amorphous phase. The amorphous phase has a much lower Curie temperature than the $\alpha$-FeSi grains [22]. Approaching the Curie temperature of the amorphous phase, the exchange coupling interaction is largely reduced, and the soft magnetic properties degrade correspondingly. The temperature dependence of the magnetic properties in the nanocrystalline $\mathrm{FeCuNbSiB}$ ribbons demonstrates that it is important to maintain an efficient exchange coupling interaction between $\alpha-\mathrm{FeSi}$ grains and the residual amorphous matrix, and it is necessary to sustain the higher Curie temperature for the application of the $\mathrm{FeCuNbSiB}$ ribbons in high temperature.

\section{Conclusions}

The microstructure and thermal magnetic behavior of the $\mathrm{FeCuNbSiB}$ ribbons annealed at different temperature are investigated in detail. All the annealed samples have crystallized partially, and their microstructure of the ribbons are consists of the residual amorphous phase, the a-FeSi crystalline phase and a little Fe3Si crystalline phase. The content of crystalline phase increase accordingly with the increase of annealing temperature. The $\alpha$-FeSi grains in the annealed ribbons are octahedral shape, which are confirms by the morphology simulation and the AFM method. The thermal behaviors of the original permeability of the ribbons are complicated, can be divided into a single Hoppkinson peak of $\alpha$-FeSi and double Hoppkinson peaks of amorphous phases and $\alpha$-FeSi phase. The Bs and Ms decrease with increasing temperature. Increasing the anneal temperature, which induce the increase of grain volume fraction, the decrease of the Ms became slower until to the curies temperature. For 
single-phase soft magnetic amorphous ribbon, the coercive force $\mathrm{Hc}$ and remanence ratio $\alpha$ have shown a continuous monotonic decline with increasing temperature. For biphase nanocrystalline ribbons, the coercive Hc and remanence ratio $\alpha$ firstly increased to a max value or a peak and then decreased to zero with the increase of temperature. The temperature at the peak of Hc is higher than that of $\alpha$, implying that there exist different mechanisms in the thermal behavior of Hc and $\alpha$.

The thermal behavior of magnetic properties are controlled by the exchange coupling interaction between the $\alpha$-FeSi grains and the intergranular amorphous phase, so researching the exchange coupling interaction of the $\alpha$-FeSi grains should consider the affect of the magnetic properties of the $\alpha-\mathrm{FeSi}$ grains, in addition to considering the affect of the amorphous phase. If the distance between $\alpha$-FeSi grains is less than the exchange-coupled grains critical size Lex, the magnetization from one grain to the other grains was carried out by penetrating through the grain boundary phase. It is important to maintain an efficient exchange coupling interaction between $\alpha$-FeSi grains and the residual amorphous matrix, and it is necessary to sustain the higher Curie temperature for the application of the $\mathrm{FeCuNbSiB}$ ribbons in high temperature by controlling the size and crystallization degree of $\alpha$-FeSi grains.

\section{Acknowledgments}

This work was supported by the National Natural Science Foundation of China (Grant no. 51401184), the Pre-Research Foundation of China General Equipment Department (Grant no. 51302010305), and the Postdoctoral Science Foundation of Ningbo Branch of China Academy of Ordnance Science (NBPJ2013-6).

\section{References}

[1]. M. Ohta, Y. Yoshizawa, High Bs nanocrystalline $\mathrm{Fe}_{84-\mathrm{x}-\mathrm{y}} \mathrm{Cu}_{\mathrm{x}} \mathrm{Nb}_{\mathrm{y}} \mathrm{Si}_{4} \mathrm{~B}_{12}$ alloys $(\mathrm{x}=0.0-1.4$, $\mathrm{y}=0-2.5$ ), Journal of Magnetism and Magnetic Materials. 321 (2009) 2220-2224.

[2]. Y.P. Liu, Y.D. Yi , W. Shao, Y.F. Shao, Microstructure and magnetic properties of soft magnetic powder cores of amorphous and nanocrystalline alloys, Journal of Magnetism and Magnetic Materials. 330 (2013) 119-133.

[3]. H.N. Farzad, B. Ali, M.F. Mohammad Kazem, The effect of quenching rate on structure and soft magnetic properties of high Bs Fe-based nanocrystalline alloys, Advanced Materials Research. 829 (2014) 78-81.

[4]. Q.Z. Zhi, B.S. Dong, W.Z. Chen, K.Y. He, Elevated temperature initial permeability study of $\mathrm{Fe}_{73.5} \mathrm{Cu}_{1} \mathrm{Nb}_{3} \mathrm{Si}_{13.5} \mathrm{~B}_{9}$ alloy, Materials Science and Engineering A. 448 (2007) 249-252.

[5]. J. Wang, Z. Wang, Y.Y. Jia, R.M. Shi, Z.P. Wen, High temperature soft magnetic properties of $\left(\mathrm{FexCo}_{1-\mathrm{x}}\right)_{73.5} \mathrm{Cu}_{1} \mathrm{Mo}_{3} \mathrm{Si}_{13.5} \mathrm{~B}_{9}(\mathrm{x}=0.5,1)$ alloys, Journal of Magnetism and Magnetic Materials. 328 (2013) 62-65.

[6]. N.V. Ershov, V.A. Lukshina, V.I. Fedorov, N.V. Dmitrieva, Y.P. Chernenkov, A.P. Potapov, Effect of Thermomagnetic and Thermomechanical Treatments on the Magnetic Properties and Structure of the Nanocrystalline Soft Magnetic Alloy $\mathrm{Fe}_{81} \mathrm{Si}_{6} \mathrm{Nb}_{3} \mathrm{~B}_{9} \mathrm{Cu}_{1}$, Physics of the Solid State. 55 (2013) 3: 508-519.

[7]. Jana Füzerová a,n, JánFüzer b, PeterKollár b, RadovanBureš c, MáriaFáberová c ,Complex permeability and core loss of soft magnetic Fe-based nanocrystalline powder cores, Journal of Magnetism and Magnetic Materials. 345 (2013) 77-81.

[8]. O. Gutfleisch, M.A. Willard, E Bruck, C.H. Chen, S.G. Sankar, J.P. Liu, Magnetic materials and devices for the 21 stcentury: stronger, lighter, and more energy efficient, Advanced Materials. 23 (2011) 821-842.

[9]. A. Hernando, T. Kulik, Exchange interactions through amorphous paramagnetic layers in 
ferromagnetic nanocrystalline, Physical Review B. 49 (1994) 7064-7070.

[10]. A. Hernando, J.M. Barandiaram, J.M. Rojo, J.C. Gomez-Sal, About the effect of pressure and volume expansion on the transition from antiferromagnetic to ferromagnetic state in some metal alloys, Journal of Magnetism and Magnetic Materials. 174 (1997) 181-184.

[11]. Y.Z. Zhang, Microstructure and temperature dependence of susceptibility of different annealed nanocrystalline $\mathrm{Fe}_{73.1} \mathrm{Cu}_{1.2} \mathrm{Nb} \mathrm{Si}_{12.5} \mathrm{~B}_{10}$ alloy, Journal of Functional Materials and Devices. 1-3 (1997) 88-93.

[12]. G. Herzer, Modern soft magnets: Amorphous and nanocrystalline materials, Acta Materialia. 61 (2013) 718-734.

[13]. N. C, Popa, The (h k l) dependence of diffraction-line broadening caused by strain and size for all Laue groups in Rietveld refinement, Journal of Applied Crystallography. 31 (1998) 176-180.

[14]. M.A. Nowroozi, H. Shokrollahi, Magnetic and structural properties of amorphous and nanocrystalline $\mathrm{Fe}_{42} \mathrm{Ni}_{28} \mathrm{Zr}_{8} \mathrm{Ta}_{2} \mathrm{~B}_{10} \mathrm{C}_{10}$ soft magnetic alloy produced by mechanical alloying, Advanced Powder Technology. 24 (2013) 1100-1108.

[15]. X.D. Fan, H. Men, A.B. Ma, B.L. Shen, Soft magnetic properties in $\mathrm{Fe}_{84-\mathrm{x}} \mathrm{B}_{10} \mathrm{C}_{6} \mathrm{Cu}_{\mathrm{x}}$ nanocrystalline alloys, Journal of Magnetism and Magnetic Materials. 326 (2013) 22-27.

[16]. F.G. Chen, Y.G. Wang, X.F. Miao, H. Hong, K. Bi, Nanocrystalline $\mathrm{Fe}_{83} \mathrm{P}_{16} \mathrm{Cu}_{1}$ soft magnetic alloy produced by crystallization of its amorphous precursor, Journal of Alloys and Compounds. 549 (2013) 26-29.

[17]. G. Herzer. Nanocrystalline soft magnetic materials. Journal of Magnetism and Magnetic Materials. 157 (1996) 133-137.

[18]. R. C. O. Handiey, Modern Magnetic Materials Principles and Applications, November, 1999, USA.

[19]. H. Ucar, J. Ipus, V. Franco, M.E. Mchenry, D. E. Laughlin, Overview of Amorphous and Nanocrystalline Magnetocaloric Materials Operating Near Room Temperature, Journal of the Minerals Metal and Materials Society. 64 (2012) 782.

[20]. K.Y. He, Magnetothermal analysis of $\mathrm{Fe}_{72.7} \mathrm{Cu}_{1} \mathrm{Nb}_{2} \mathrm{~V}_{1.8} \mathrm{Si}_{13.5} \mathrm{~B}_{9}$ alloy, Acta metallurgica sinica. 33 (1997) 12: 356-359.

[21]. S. Ji, The microstructure and properties of Fe-based soft magnetic alloy composed of ultrafine grain, University of Science and Technology Beijing, PhD Theis, 1994

[22]. J.F. Loffler, J.P. Meier, B. Doudin, J.P. Ansermet, W. Werner, Random and exchange anisotropy in consolidated nanostructured $\mathrm{Fe}$ and $\mathrm{Ni}$ : role of grain size and trace oxides on the magnetic properties, Physical Review B. 57 (1998) 2915-2924. 